\title{
CARIOTIPOS Y ESTUDIOS MEIOTICOS EN VARIAS ESPECIES DE PIRIQUETA (TURNERACEAE)
}

por GRACIELA INES LAVIA' y AVELIANO FERNANDEZ ${ }^{2}$

\section{Summary}

The karyotypes of six species of the genus Piriqueta are described for the first time. The basic chromosome number is $\mathrm{x}=7$. Four species are diploid, $P$. cistoides ssp. cistoides and $P$. racemosa with $2 \mathrm{n}=2 \mathrm{x}=14=14 \mathrm{~m}$; $P$. australis $2 \mathrm{n}=2 \mathrm{x}=14=12 \mathrm{~m}+2 \mathrm{sm} ; P$. duarteana, $2 \mathrm{n}=2 \mathrm{x}=14=12 \mathrm{~m}$ $+2 \mathrm{st}$; $P$. rosea, with two cytotypes, diploid $(14 \mathrm{~m})$ and tetraploid $(28 \mathrm{~m})$ and $P$. taubatensis, a polyploid species, with $2 n=6 x=42=38 m+4 s m$.

The diploid species, $P$. cistoides, $P$. racemosa, $P$. australis, $P$. ochroleuca and $P$. sidifolia var. multiflora, present regular meiosis, with 7 II; while the pairing in $P$. taubatensis shows multivalents, suggesting that this species is a segmental allohexaploid.

The karyotypes are different in ploidy levels, symmetry, number of metacentrics, submetacentrics and subtelocentrics, and number and position of secondary constrictions. In some of the species there is differential staining intensity in the bivalents.

\section{Introducción}

Piriqueta y Turnera son los géneros más importantes dentro de la familia Turneraceae. Piriqueta cuenta con 42 especies, de las cuales una es africana y las restantes se distribuyen desde el sur de EEUU de Norteamérica hasta el norte de Argentina.

\footnotetext{
'Becaria deI CONICET Instituto de Butánica del Nordeste (UNNE-CONICET). C.C. 209 - 3400 Corrientes, Argentina.

${ }^{2}$ Miembro de la Carrera del Investigador Científico, CONICET. Instituto de Botánica del Nordeste (UNNE-CONICET). C.C. 209 - 3400 Corrientes, Argentina.
} 
Los antecedentes citológicos existentes en el género Piriqueta son muy escasos, se conocen los trabajos de Lewis et al. (1962) y de Ordunff (1970). En el trabajo publicado por Fernández (1987) se da a conocer el número cromosómico de ocho especies diploides, dos tetraploides y una hexaploide, lo que representa el $25 \%$ de las especies del género. Hasta la fecha no se han llevado a cabo estudios detallados de los cariotipos.

En el presente trabajo se da a conocer por primera vez el número cromosómico de $P$. sidifolia var. multiflora, y de accesiones nuevas de $P$. australis, $P$. cistoides ssp. cistoides, $P$. duarteana var. duarteana. Se llevó a cabo el análisis cariotípico y meiótico de $P$. australis, $P$. cistoides, $P$. racemosa y $P$. taubatensis, el cariotípico de $P$. duarteana y $P$. rosea, y el meiótico de $P$. sidifolia y $P$. ochroleuca.

\section{Material y métodos}

El material estudiado figura en la Tabla I, el mismo se halla depositado en el herbario del Instituto de Botánica del Nordeste (CTES).

Los estudios mitóticos se realizaron en meristemas de raíces provenientes de semillas germinadas y gajos puestos a enraizar, que se sometieron a los siguientes pasos:

a- pretratamiento con 8 -hidroxiquinoleína $(0,002 \mathrm{M})$ durante 3 horas a temperatura de laboratorio,

b- fijación en etanol absoluto-ácido acético (3:1) durante 24 horas en refrigerador,

c- conservación en alcohol $70 \%$ en refrigerador,

d- coloración con la técnica de Feulgen, que consiste en: hidrólisis con $\mathrm{HCl}(1 \mathrm{~N})$ a $60^{\circ} \mathrm{C}$ durante $8^{\prime}$, transferencia a reactivo de Schiff $15^{\prime}$ a $30^{\prime}$ en oscuridad,

e-montaje en orceína al $2 \%$.

La nomenclatura utilizada en la descripción de los cariotipos es la recomendada por Levan et al. (1964). La morfología de los cromosomas fue determinada utilizando el índice centromérico (brazo corto x 100 / largo total del cromosoma). Se distinguieron cromosomas: metacéntricos $(\mathrm{m})=$ 
50-37,5, submetacéntricos $(\mathrm{sm})=37,5-25$ y subtelocéntricos $(\mathrm{st})=25-$ 12,5 .

La estimación de la asimetría de los cariotipos se realizó por medio de los índices de asimetría intra- e intercromosómica $\mathrm{A}_{1}$ y $\mathrm{A}_{2}$ (Romero Zarco, 1986). El valor de estos índices varía de 0 a 1, la simetría es mayor cuando el valor se acerca a 0 .

$$
\begin{aligned}
& \sum \frac{\mathrm{b}}{\mathrm{B}} \mathrm{A} \\
& \mathrm{A}_{1}=1-\frac{\mathrm{B}}{\mathrm{n}} \quad \mathrm{A} \text { brazo corto } \\
& \mathrm{B}=\mathrm{x} \text { brazo largo } \\
& \mathrm{n}=\text { número de cromosomas } \\
& \mathrm{S}=\text { desvío estandar } \\
& \mathrm{x}=\text { media de la long. total } \\
& \text { de crom. } \\
& \Sigma=\text { sumatoria }
\end{aligned}
$$

En los estudios meióticos, a partir de la fijación se siguieron los mismos pasos que en los mitóticos.

Los nucleolos fueron teñidos siguiendo la técnica de Howell y Black (1980) modificada:

1 - pretratamiento en 8-oxiquinoleína 0,002 M durante 3 horas a temperatura de laboratorio,

2- fijación en etanol absoluto-ácido acético (3:1), entre 20 a 70 horas a $4^{\circ} \mathrm{C}$,

3- hidrólisis $30^{\prime \prime}$ en ácido clorhídrico $1 \mathrm{~N} \mathrm{a} 60^{\circ} \mathrm{C}$,

4- aplastado en ácido acético $45 \%$,

5- desprendimiento del cubreobjetos en alcohol absoluto,

6 - secado y tinción con nitrato de plata al $50 \%$.

\section{Resultados}

Las características cromosómicas de las distintas especies, que se muestran en las Tablas 2, 3, 4 y en las Figuras 1 y 2, se describen a continuación. 
Tabla 1: Material estudiado

Especie

nov. comb. ined.

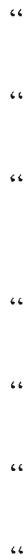

P. cistoides subsp. cistoides

(L.) Griseb.
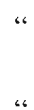

“

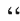

« 2n $\quad N^{\circ}$ de col, y procedencia

14 De Pompert 24, Argentina, Corrientes, Santa Ana.

14 Ferraro 2969, Argentina, Corrientes, Ituzaingó. \&

14 Pott et al. 1099, Brasil, MS, Corumbá, Morro Sao Domingos.

14 Pott et al. 1155, Brasi], MS, Corumbá, Estrada do Porto da Manga-Banda Alta. a $22 \mathrm{~km}$ de Corumbá.

14 Vanni et al. 1277, Paraguay, Presidente Hayes, Puerto Militar.

14 Zardini et al. 3769, Paraguay, Cordillera, Cerro Tobatí.

14 Zardini 26119, Paraguay, Cordillera, Cerro Tobatí.

14 Zardini 27453, Paraguay, Cordillera, Cerro Tobatí.

14 Jiménez 9081, Rep. Dominicana, Santiago, Rincón de Piedra.

$\&$

14 Krapovickas \& Cristóbal 42760, Brasil, MG, $17 \mathrm{~km}$ W de Itapagipe.

14 Krapovickas \& Cristóbal 43087, Brasil, MT, Transpantaneira, $15 \mathrm{~km}$ de Poconé, Puesto IBDF.

14 Beck 17056, Bolivia, Pando, Nicolás Suárez, Cobija.

14 Arbo et al. 3000, Brasil, SP, Rodovia SP-330, vía Anhanguera, $10 \mathrm{~km} \mathrm{~S} \mathrm{del}$ río Grande.

14 Kirizawa 750, Brasil, PR, Guaira Sete Quedas. 
P. duarteana (Cambess.)

Urban var. duarteana

P. ochroleuca Urban

$P$. racemosa (Jacq.) Sweet

$P$. rosea (Cambess.) Urban

“

P. sidifolia (Cambess.)

Urban var. multiflora Urban

P. taubatensis (Urban) Arbo “
14 Hatschbach et al. 56558, Brasil, BA BR-030, 7 km Sur de Caetité. $+@$

14 Krapovickas 38735, Brasil, PI, Gilbués, BR-135.

14 Krapovickas \& Cristóbal 42890, Brasil, MG, Pirapora.

14 Krapovickas \& Cristóbal 42893, Brasil, MG, BR-365, 12 Km SW de Pirapora.

14 Bordas 4048, Paraguay, Cordillera, Caacupé (Barrio Kennedy).

14 Krapovickas 38807, Brasil, BA, $1 \mathrm{~km} N$ de Baixa Grande.

14 Krapovickas 34405, Brasil, MS, $38 \mathrm{Km}$ W de Ribas do Rio Pardo.

28 Krapovickas 34428, Brasil, MS, $85 \mathrm{~km}$ de Campo Grande, camino a Aquidauana.

42 Arbo 2463, Brasil, RS, $35 \mathrm{~km}$ al E de Rosario do Sul. \#

42 Arbo 2544, Argentina, Misiones, Candelaria.

\& análisis cariotípico y meiótico

\# análisis cariotípico

+ análisis meiótico

* recuentos en nuevas accesiones

(@) nuevos recuentos 
P. cistoides ssp. cistoides: $2 \mathrm{n}=14=14 \mathrm{~m}$ (Fig. 1 A), publicada como $P$. cistoides por Fernández (1987). Posee los cromosomas más pequeños de las especies estudiadas, el promedio es de $1,01 \mu \mathrm{m}$ variando desde 0,69 a 1,39 $\mu \mathrm{m}$. El primer par presenta constricción secundaria en el brazo corto.

P. racemosa: $2 \mathrm{n}=14=14 \mathrm{~m}$ (Fig. 1B), la longitud de sus cromosomas varía entre 0,85 ! $\mathrm{m}$ y $1,61 \mu \mathrm{m}$. Esta especie posee $14 \mathrm{~m}$ igual que la anterior, pero no se ha observado satélite.

Tabla 2: Características cromosómicas

\begin{tabular}{lccccc}
\hline Especie & 2n & $\begin{array}{c}\text { Fórmula } \\
\text { cariotípica }\end{array}$ & $\begin{array}{c}\text { Tamaño } \bar{x} \text { por } \\
\text { crom. en } \mu \mathrm{m}\end{array}$ & $\begin{array}{c}\text { Rango de var. } \\
\text { en } \mu \mathrm{m}\end{array}$ & $\begin{array}{c}\text { Ubic. del } \\
\text { satélite }\end{array}$ \\
\hline$P$. cistoides & 14 & $14 \mathrm{~m}$ & 1,01 & $0,69-1,39$ & B.C. par 1 \\
$P$. racemosa & 14 & $14 \mathrm{~m}$ & 1,14 & $0,85-1,61$ & - \\
$P$. australis & 14 & $12 \mathrm{~m}+2 \mathrm{sm}$ & 1,13 & $0,75-1,57$ & B.L. par 7 \\
$P$. duarteana & 14 & $12 \mathrm{~m}+2 \mathrm{st}$ & 1,33 & $1,02-1,99$ & B.C. par 6 \\
$P$. rosea & 14 & $14 \mathrm{~m}$ & 1,67 & $1,11-2,91$ & B.L. par 2 \\
$P$. rosea & 28 & $28 \mathrm{~m}$ & 1,44 & $0,91-2,09$ & B.L. par 3-4 \\
$P$. taubatensis & 42 & $38 \mathrm{~m}+4 \mathrm{sm}$ & 1,44 & $0,97-2,37$ & B.C. par 1-2 \\
\hline
\end{tabular}

Tabla 3: Long. total del complemento (LTC), promedio del índice centromérico $(\bar{x} \mathrm{IC})$, índice de asimetría intracromosómica $\left(\mathrm{A}_{\mathrm{1}}\right)$, índice de asimetría intercromosómica $\left(\mathrm{A}_{2}\right)$

\begin{tabular}{llllll}
\hline Especie & 2n & LTC & $\bar{x}$ IC & $\mathrm{A}_{1}$ & $\mathrm{~A}_{2}$ \\
\hline$P$. cistoides & 14 & 14,08 & 44,87 & 0,20 & 0,25 \\
$P$. racemosa & 14 & 15,92 & 45,86 & 0,20 & 0,25 \\
$P$. australis & 14 & 15,78 & 45,05 & 0,17 & 0,31 \\
$P$. duarteana & 14 & 18,66 & 42,44 & 0,24 & 0,28 \\
$P$. rosea & 14 & 23,42 & 45,62 & 0,24 & 0,37 \\
$P$. rosea & 28 & 40,32 & 45,88 & 0,15 & 0,23 \\
$P$. taubatensis & 42 & 60,48 & 45,27 & 0,17 & 0,27 \\
\hline
\end{tabular}


Tabla 4: Promedio \pm E.S. y variación de las asociaciones cromosómicas en Metafase I

\begin{tabular}{|c|c|c|c|c|c|c|c|c|}
\hline Especie & $2 n$ & I & II & III & IV & $\mathrm{V}$ & VI & CMP \\
\hline P. cistoides & 14 & & 7 & & & & & 30 \\
\hline P. racemosa & 14 & & 7 & & & & & 23 \\
\hline P. australis & 14 & & 7 & & & & & 8 \\
\hline P. ochroleuca & 14 & & 7 & & & & & 12 \\
\hline P. sidifolia & 14 & & 7 & & & & & 45 \\
\hline \multirow[t]{3}{*}{ P. taubatensis } & 42 & 2,17 & 9,67 & 1.83 & 1,50 & 0,50 & 1,00 & 6 \\
\hline & & $\pm 0,60$ & $\pm 1,97$ & $\pm 0,48$ & $\pm 0,62$ & $\pm 0,34$ & \pm 0.37 & \\
\hline & & $0-4$ & $6-17$ & $0-3$ & $0-4$ & $0-2$ & $0-2$ & \\
\hline
\end{tabular}

$P$. australis: $2 \mathrm{n}=14=12 \mathrm{~m}+2 \mathrm{sm}$ (Fig. 1C), citada bajo la denominación de $P$. viscosa Griseb. Fernández (1987). Esta especie se diferencia de las dos anteriores en un par submetacéntrico que lleva macrosatélite (Fig. 2 A). La longitud de los cromosomas oscila de 0,75 a $1,57 \mu \mathrm{m}$.

P. duarteana var. duarteana: $2 \mathrm{n}=14=12 \mathrm{~m}+2$ st (Fig. 1D), publicada como P. aff. duarteana por Fernández (1987). La longitud de sus cromosomas oscila entre 1,02 y 1,99 $\mu \mathrm{m}$. Esta especie es la única que posee un par de cromosomas subtelocéntricos; además se observa constricción secundaria en el brazo corto del par 6 (Fig. 2B).

P. rosea: (Fig. 1E), posee dos citotipos, el diploide $2 \mathrm{n}=14=14 \mathrm{~m}$ ( $P$. serrulata Urban en Fernández, 1987) y el tetraploide $2 \mathrm{n}=28=28 \mathrm{~m}$ ( $P$. rosea var. occidentalis en Fernández, 1987). En ambos citotipos los cromosomas son todos metacéntricos, observándose que el par 1 se destaca del resto por su tamaño. El par 2 posee macrosatélite en el brazo largo. En la Fig. 1 no se ha representado el citotipo tetraploide porque es igual al diploide, repitiéndose cada cromosoma cuatro veces.

P. taubatensis: $2 \mathrm{n}=42=38 \mathrm{~m}+4 \mathrm{sm}$ (Fig. $1 \mathrm{~F}$ ), tiene 2 pares de cromosomas con satélite en el brazo corto (Fig. 2C). El tamaño de los cromosomas varía entre 0,97 y $2,37 \mu \mathrm{m}$. 
A

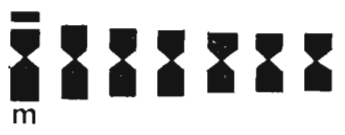

$\mathrm{B}$

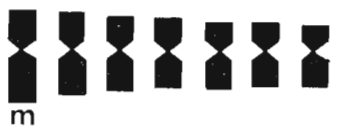

C

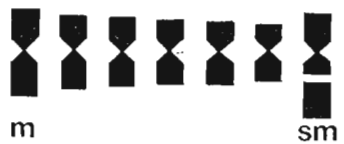

1

D

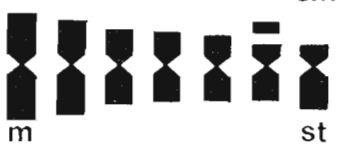

E

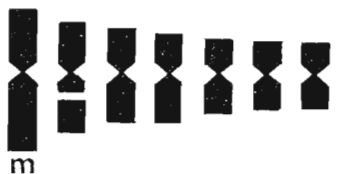

t

m

$\mathrm{F}$

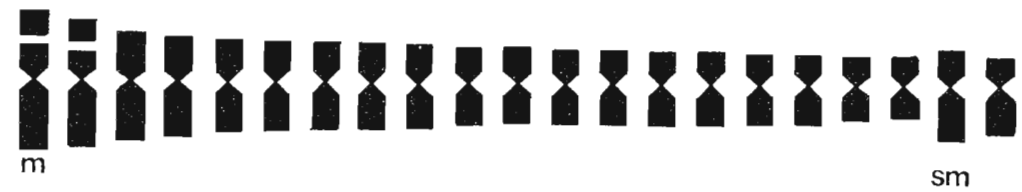

Fig. 1.- Idiogramas. A, P. cistoides; B, P. racemosa; C, P. australis; D, P. duarteana; E, $P$. rosea; F, P. taubatensis. Escala $=2,40 \mu \mathrm{m}$.

Fig. 2.- Mitosis, meiosis y nucleolos. A, P. australis, $2 \mathrm{n}=14 ; \mathrm{B}, P$. duarteana, $2 \mathrm{n}=$ 14; C, P. taubatensis, $2 \mathrm{n}=42$; las flechas indican los satélites. D, P. racemosa, $7 \mathrm{II}$; E, $P$. sidifolia, 7 II, posee 2 de mayor tamaño (señalados); F, P. taubatensis, II +7 II +3 III +2 IV +2 VI (señalados). G, P. taubatensis, nucleolos en interfase, se indica con flecha la célula conteniendo 4 nucleolos. 

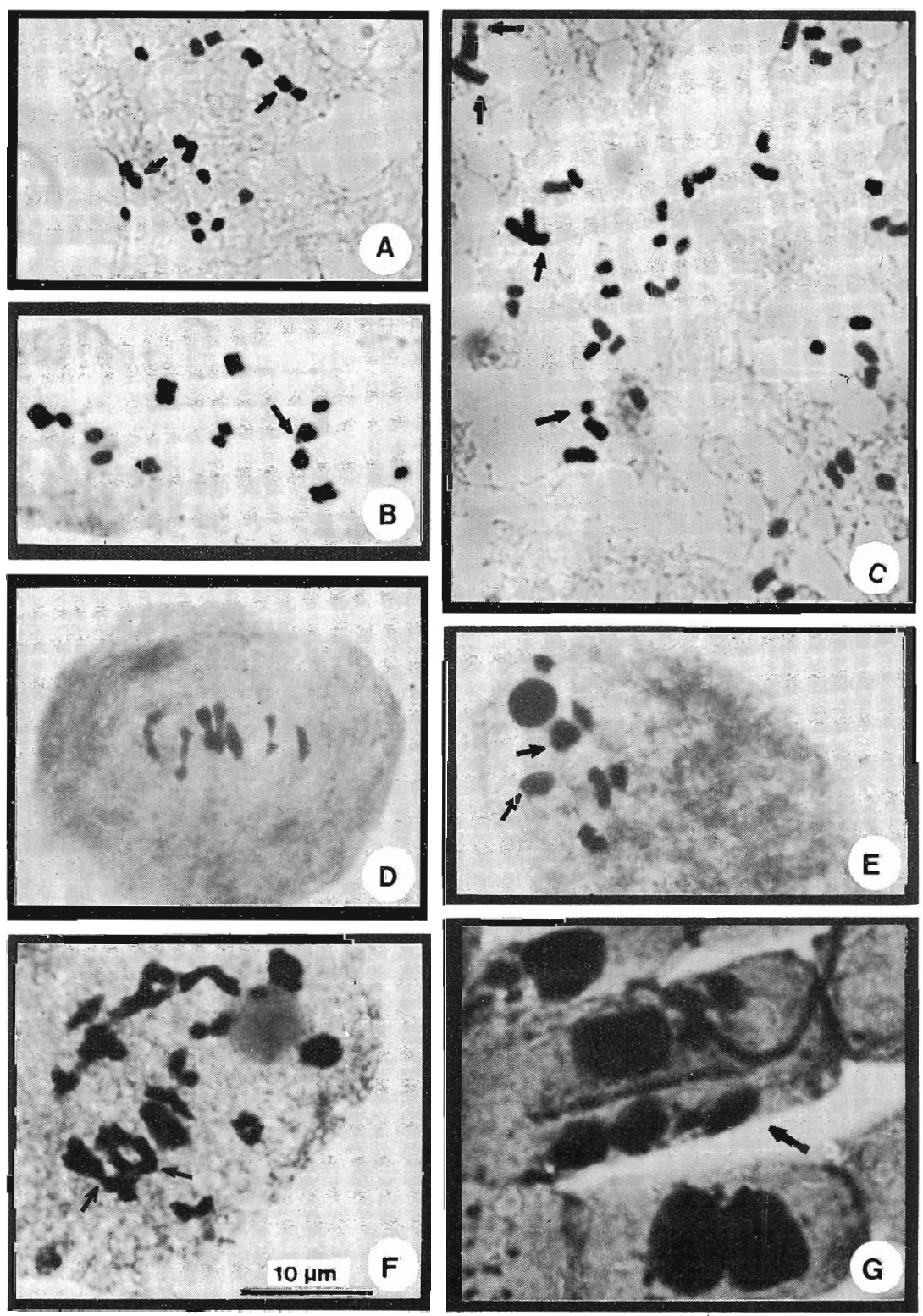
La meiosis de las especies diploides es regular, formando 7 II (Tabla 4). En la Fig. 2 D se observan los 7 bivalentes en $P$. racemosa.

En $P$. ochroleuca y $P$. sidifolia (Fig. 2E) se observaron 2 bivalentes de coloración más intensa y de mayor tamaño que el resto; en $P$. racemosa se halló un solo bivalente con estas características, que correspondería al par 1; en cambio, en $P$. cistoides los 7 bivalentes presentaron igual coloración.

En $P$. taubatensis, $2 \mathrm{n}=6 \mathrm{x}=42$, hasta el presente se analizaron únicamente 6 CMP, presentando un promedio de bivalentes muy bajo $(9,67)$ y un máximo de 17 II por célula (Tabla 4); el promedio de sexivalentes fue de 1.00, hallándose hasta 2 sexivalentes por célula (Fig. 2F). Estos datos estarían indicando que se trata de un alohexaploide segmentario.

\section{Discusión}

Hasta el momento se ha hecho el análisis detallado de los cariotipos de 6 especies, 4 de ellas diploides ( $P$. cistoides ssp. cistoides, $P$. racemosa, $P$. australis y $P$. duarteana), 1 hexaploide ( $P$. taubatensis), y 1 con dos citotipos, diploide y tetraploide $(P$. rosea).

Según la clasificación de Lima de Faría (1980), los cromosomas de las especies de Piriqueta presentadas en este trabajo estarían comprendidos entre las categorías de rudimentarios y pequeños, ya que algunos miden menos de una micra.

Todas las especies estudiadas poseen al menos un par de cromosomas con satélite, excepto $P$. racemosa donde no fue posible observarlos. $P$. australis presenta los satélites de mayor tamaño (Fig. 1C).

Los cariotipos son homogéneos, ya que la variación del tamaño de los cromosomas es gradual. Los cromosomas son metacéntricos, excepto en $P$. australis que posee un par sm, $P$. duarteana un par st y $P$. taubatensis dos pares sm (Fig. 1, Tabla 2).

Tanto los índices centroméricos como los de asimetría $A_{1}$ y $A_{2}$, están indicando que estos cariotipos son simétricos (Tabla 3); $P$. duarteana es la más asimétrica por la presencia de un par subtelocéntrico. 
$P$. cistoides y $P$. racemosa poseen características cromosómicas muy semejantes entre sí, diferenciándose por la presencia de satélite en $P$. cistoides (Fig. 1 A y B). Sus índices de asimetría $\left(A_{1}\right.$ y $\left.A_{2}\right)$ coinciden totalmente, pero no el índice centromérico (Tabla 3).

El citotipo diploide de $P$. rosea posee los 14 cromosomas metacéntricos, como en $P$. cistoides y $P$. racemosa, diferenciándose por su mayor tamaño (1,67 micras de promedio), mientras que las otras dos especies poseen 1,01 y 1,14 micras respectivamente. Además, el satélite de $P$. rosea es de mayor tamaño que el de $P$. cistoides y está ubicado en el brazo largo del par 2. En cuanto al citotipo tetraploide de $P$. rosea, los cromosomas observados en metafases mitóticas se pueden agrupar de a 4 , lo que indicaría que se trata de un autotetraploide. Lewis (1967), había llegado a la conclusión de que los diploides y sus derivados autotetraploides constituyen una sola especie, porque hay continuidad genética entre ellas, Arbo y Fernández (1983), llegan a la misma conclusión para Turnera subulata.

En $P$. taubatensis se observa que los pares 1 y 2 son muy semejantes, teniendo en cuenta la longitud y la presencia de satélite en el brazo corto; como así también son similares entre sí los pares 20 y 21 , ambos sm, (Fig. 1F); probablemente se trataría de cromosomas homeólogos ya que en la meiosis se observaron cuadrivalentes. Mediante la técnica de AgNOR, se comprobó por la presencia de 4 nucleolos (Fig. 2G), que los dos pares con satélite son organizadores nucleolares.

Relacionando la longitud de estilo/estambre con el nivel de ploidía de las especies estudiadas, se observó que la única especie homostila y autocompatible es diploide; en cambio las heterostilas y autoincompatibles pueden ser diploides o poliploides. En Turnera ocurre lo contrario, pues las especies heterostilas autoincompatibles son diploides, mientras las homostilas autocompatibles son hexaploides (Barrett, 1978; Barrett y Shore, 1980).

Piriqueta está muy relacionado con el género Turnera, la serie Salicifoliae es, por su morfología floral, la más afín (Arbo, 1986). Esta afinidad se presenta también citológicamente, ya que el número básico y las 
características cromosómicas de Turnera weddelliana, perteneciente a la serie Salicifoliae (Solís Neffa y Fernández, 1992), son similares a las especies del género Piriqueta estudiadas hasta la fecha.

Piriqueta no presenta la variación morfológica de Turnera, sin embargo se observan diferencias citológicas entre los cariotipos analizados. Los mismos difieren entre sí en cuanto al nivel de ploidía, la asimetría del cariotipo, el número de metacéntricos, submetacéntricos y subtelocéntricos, y la presencia y posición de las constricciones secundarias.

\section{Agradecimientos}

Agradecemos a la Lic. María Mercedes Arbo por proporcionar e identificar el material y por leer en forma crítica el manuscrito.

\section{Bibliografía}

Arbo, M. M. 1986. Paraguay, centro importante de especiación en las Turneráceas. Candollea 41: 212-218.

- Revisión del género Piriqueta. En prensa en Flora Neotrópica.

- y A. Fernández. 1983. Posición taxonómica, citología y palinología de tres niveles de ploidía de Turnera subulata Smith. Bonplandia 5 (23): 212-226.

Barrett, S.C.H. 1987. Variation and evolution of breeding systems in the Turnera ulmifolia L. complex (Turneraceae). Evolution, 41 (2): 340354.

Fernández, A. 1987. Estudios cromosómicos en Turnera y Piriqueta. Bonplandia 6 (1): 1-21.

Howell, W.M. \& D.A. Black. 1980. Controlled silver staining of nucleolus organizer region with a protective colloidal developer: a 1 step method. Experientia 36:1014-1015.

Levan, A.; K. Fredga \& A. A. Sandberg. 1964. Nomenclature for centromeric position on chromosomes. Hereditas 52: 201-220.

Lewis, H. 1967. The taxonomic significance of autopolyploidy. Taxon 16: 267-271. 
-; H. L. Stripling y R. G. Ross. 1962. Chromosome numbers for some Angiosperms of the southern United States and Mexico. Rhodora 64 (758): 147-161.

Lima De Faría, A. 1980. Classification of genes, rearrangements and chromosomes according to the field. Hereditas 93: 1-46.

Ordunff, R. 1970. Relationships in the Piriqueta caroliniana-P. cistoides complex (Turneraceae). J. Arnold Arbor. 5: 491-498.

Romero Zarco, C. 1986. A new method for estimating karyotype asymmetry. Taxon 35: 526-530.

Solís Neffa, V. y A. Fernández. 1992. Estudios cromosómicos en especies de Turnera del Paraguay. Resúmenes de las III Jornadas de Biología del Paraguay. 